\title{
A Conversation with Helen Mayberg
}

\author{
InTERVIEWER: SÉBastien Thuault \\ Senior Editor, Nature Neuroscience
}

\begin{abstract}
Helen Mayberg is a Professor in the Departments of Neurology, Neurosurgery, Neuroscience, and Psychiatry at the Icahn School of Medicine and Director of the Center for Advanced Circuit Therapeutics at Mount Sinai in New York City.
\end{abstract}

Sébastien Thuault: We all know individuals with depression. Could you do a bit of a presentation on this condition? What are the therapies that are available to the patient population at this time?

Dr. Mayberg: Depression is a very common psychiatric condition, one of the most common that we have. It's diagnosed by an interview, not by a laboratory test or a scan. It's a condition that's characterized by a disturbance in one's mood, generally a profoundly and distressing low mood that's accompanied, in most cases, by a sense of not feeling pleasure: anhedonia. It has an admixture of many other symptoms that affect our drives-like sleep, or appetite, or one's libido - and activities, as well as interests and thought. Thinking can get slow or confused. People feel like doing less. They may feel anxiety. They may have sensations in the body that, at their core, are disturbances in drive states, emotion, and putting thought to action.

Despite being extremely common and accounting for much of the world's disability, the good news is that there are many treatments, and have been. There are a number of evidence-based psychotherapies like interpersonal psychotherapy or cognitive behavioral therapy that can be quite effective. There's a multitude of medications and have been since the late ' $50 \mathrm{~s}$, heralded now by the serotonin reuptake inhibitors, but also drugs like tricyclics or mixed serotonin and norepinephrine drugs. We have new medications like ketamine that are being tested that are not even of the old classes. And we have somatic treatments, like using magnetic therapies like TMS [transcranial magnetic stimulation]. Most importantly, the best treatment that there is is actually electroconvulsive therapy, but it's generally reserved for people who are extremely ill and have not responded to medication or psychotherapy.

Sébastien Thuault: You actually explore another approach to therapy, which is deep brain stimulation. From what I understand, this is something that was inspired by the use of this approach for motor disorders like Parkinson's disease. Why don't you tell us about your research in this area, perhaps starting by how did you decide initially to explore this avenue to provide patients with relief?
Dr. Mayberg: Going back, I originally trained as a neurologist at Columbia and started my research as a postdoc in the radiology department at Johns Hopkins. There I had the opportunity to work on chemical mapping with PET [positron emission tomography] scanning, predominantly examining opiate receptors. I actually studied epilepsy in my first experiments but was introduced to a team that was studying depression in neurological disease. We started to map resting regional glucose metabolism in those depressed patients - using patients with different neurological diagnoses with and without depression - and a pattern of consistent abnormalities across different groups of depressed patients emerged. When we had some confidence that there was a signal from the scans of depressed patients with stroke, Parkinson's disease, and Huntington's disease, we moved to studies of primary depression and then to studies of the effects of treatments to characterize how regional metabolism changed as patients recovered.

We fell on this one region, that actually wasn't abnormal to start with, but that showed down-regulated metabolic activity when people successfully recovered with a typical SSRI medication. We saw the same pattern with placebo responders and in response to various other classes of antidepressant medication; we also saw changes in this region with psychotherapy. We started to hone in on not just this region but the brain-wide pattern of treatment-specific changes in depression and depression recovery or nonrecovery, building a virtual network map, a depression circuit so to speak. The subcallosal cingulate Area 25-using the Brodmann characterization - seemed to be vitally important to this dynamic circuit: if it didn't change, you didn't get well. The next set of studies focused on the details of this depression network and this evolving mood circuit. We were looking at these systems in different patient groups, with not just PET but also with fMRI, and at not just at rest but with various mood- and depression-specific tasks.

When I moved to Toronto, I was introduced very early on to the neurosurgeons working with the Parkinson's group. The Parkinson's disease field had already established a strategy for treating severe PD by directly tuning basal ganglia circuits with deep brain stimulation. This strategy built directly on a long history of work on the

(C) 2018 Mayberg. This article is distributed under the terms of the Creative Commons Attribution-NonCommercial License, which permits reuse and redistribution, except for commercial purposes, provided that the original author and source are credited. 
brain's basal ganglia motor circuits, mostly established in animal studies. Not coincidentally, we had actually modeled a lot of our original neurological depression studies on these same basal ganglia circuits, so I revisited the basal ganglia story. The DBS strategy for Parkinson's disease targeted a well-established circuit. We had a putative "depression" circuit as well, albeit clearly less well defined and without the animal models. We knew we wanted activity in our circuit and particularly Area 25 to be turned down or blocked. We knew if you couldn't talk it or drug it or shock it into normal functioning, maybe we should leverage this DBS technology to down-regulate the activity directly. We knew where to we wanted to implant, as they did. They used high frequency and blocked local activity. We wanted to do the same.

Sébastien Thuault: Which fit with your hypothesis.

Dr. Mayberg: At that time, it did fit our hypothesis, so we tried it. Over time, it turned out it was the wrong hypothesis. The good news is that we had a clinical signal.

Sébastien Thuault: Can you tell us about this particular technique of inserting electrodes? How local is the stimulation? What it does to the area, and perhaps beyond?

Dr. Mayberg: This procedure is a standard neurosurgical procedure. In the operating room, the neurosurgeon makes a small opening in your skull about the size of a nickel, one on each side right at the top of your head. Then you are placed in a stereotactic frame that provides a communication link between the MRI [magnetic resonance imaging] scan and the surgical navigation system that allows the neurosurgeon to precisely implant the DBS lead at the desired location. The DBS lead is about the size of a strand of vermicelli; it's about a millimeter-and-a-half in diameter. The electrode is placed under stereotaxic guidance with the patient awake to the target in the brain on each side.

Sébastien Thuault: This is an awake surgery?

Dr. Mayberg: It can be done awake or asleep. Generally, in Parkinson's patients implanted while awake you're able to record and to know when you've reached the target because of the characteristic physiological firing patterns of parts of the subthalamic nucleus or adjacent areas you are targeting. Physiologists have learned what the signal looks like for the various basal ganglia locations they utilize, and they can test how this signal and the motor symptoms change with stimulation delivered right there in the $\mathrm{OR}$, immediately confirming they are in the right place. We wanted patients awake so that we could record and see if we also could detect some sort of localizing physiological signals. It turns out there wasn't anything really helpful, but with the imaging guidance and information we had from our prior PET and MRI studies we were able to make a reasonable approximation of the target. Once we guided the electrode to where we wanted it to be, we used behavior...

Actually, to be really transparent about it, we didn't know what was going to happen. Part of studying the initial patients awake was because it could have gone exactly the opposite of what we wanted, but by having patients awake we could turn it on, we could test different parameters. We really weren't ever expecting anything to happen immediately. We mostly wanted to make sure nothing bad happened.

Sébastien Thuault: You collaborate with a neurosurgeon for these approaches. Were you in the room with the team during these first experiments? Tell us about the first time.

Dr. Mayberg: The first time is always the most memorable of all events. It's also the most nerve-wracking, because you go from an idea, to getting all the permissions, to actually finding a patient that's agreeable, to then moving to an operating room. You move from the thought experiment to reality. The patient was awake, and once the implants were done-which was quite straightforward; Dr. Lozano's a very experienced functional neurosurgeon - that patient was basically prepared that "this is the point that your job is to tell us if you notice anything," because our intent was just to make sure that giving stimulation in this location was safe.

For me, knowing the intricacies of the neuroanatomy of this location, I was concerned that we would have some instability in blood pressure, or pulse, or drive functions, because of the connections to the hypothalamus and the insula. So everyone was poised because all you do is start with low current, you go to turn it up, you're just watching. You have monitors, but basically you have the patient. The patient wants to get better, but on the other hand doesn't know what's going to happen, and we don't know what's going to happen, so the instruction is "You just need to tell me if you notice anything, period. Good, bad, indifferent, or nothing, there's no right answer."

So you start. The electrode has got four places you can stimulate. It makes sense to start at the lowest one with the lowest current, and just slowly turn it up. Nothing happens, so we move to the next contact. Start low current, turn it up, nothing happens. "Okay, keep going." Go to the third contact, turn it on, turn it up. We get to an intermediate amount of current and the patient goes, "Did you just do something?" "Why do you ask?" "Well, I suddenly feel incredibly calm." I said, "Well, maybe you're just getting more relaxed." She goes, "No, what are you doing?"

We turned it up a little more, and she goes, "Well, that's interesting, the void just lifted." And I went, "What's a void?" It's not a term that's in the psychiatric nomenclature, and certainly not on the clinical checklist. As she tried to describe what had happened to her, that was that moment when we realized that maybe there were things about depression that could happen very rapidly. That was not our expectation; we were in that OR to see that we didn't do anything bad. She began to describe this calmness that she hadn't remembered in a long time. As she tried, we went into "Is it like Valium? Are you falling asleep? What are the choices of calm?" It was clear that this was some internal state where something very negative had just been released.

Then we turned it off, and she said, "Oh, maybe I was just imagining it." Then we turned it on again, and it lifted 
again, and we tried to get her to describe it. She got very frustrated, and she goes, "You're trying to ask me to describe the difference between a laugh and a smile." She explained that it was like the first day of springand this was in Toronto-where you go outside, it's still freezing, there's snow on the ground, and you see a crocus popping through the snow. You can just imagine that spring is coming.

That was in May of 2003, but I can still remember that sensation of being so worried something would go wrong, and this would be my fault for starting this, and then listening to a woman that literally had chains come off. It was so subtle. It was not laughter. It was not hypomania. It was this subtle... there was a sense of relief. Because the movement disorder people routinely — when you got in the right spot and turned the current up — could watch a tremor immediately halt, I think we all were wondering what we were watching. But we wanted to get the other electrode in, retest, and then get out of the operating room, figuring we would get similar phenomena if we did it outside the operating room. Unlike that first case, we did not always see that early on. But over time, that thing seen in that first patient has become a very critical part of the experiments.

Sébastien Thuault: It sounds like it was really an amazing experience for you also as a researcher, as a clinician. What happened after? You tried to scale up your efforts and work with different cohorts. Why don't you tell us a little bit about the results of this?

Dr. Mayberg: Like any experiment, it's not an $n$ of 1 , so we did six, then published. We got four out of six better. We expanded to 20 , got $60 \%$ better. And then industry became interested, in that this may be something to develop commercially. They ran their own pilot studies, and got not quite as good a result, but actually a good enough signal to be able to plan a clinical trial. Meantime, I moved from Toronto to Emory. I had a new team, a new surgeon, a new psychiatrist. We worked independently, separate from both Toronto and the clinical trial, and we were very interested in mapping out where exactly we were and how to understand the circuit better, not just the spot. So there was a divergence in the goals of the various teams. The industry trial went with the original method to test efficacy in a large cohort of patients. At Emory, we focused on small experimental cohorts with experiments designed to understand responder-nonresponder differences. By retrospectively defining the necessary white matter bundles impacted by successful DBS, we were able prospectively predict the acute intraoperative behavioral effects as well as the optimal site for long-term stimulation. Using this iterative approach, we revised our surgical targeting method that utilizes a white-matter blueprint to customize the procedure for each individual patient.

The BROADEN Study that was run and sponsored by St. Jude Medical Neuromodulation, the device company, designed a 6-month active versus sham randomized control clinical trial that at the halfway mark with 90 subjects, didn't show a significant difference between the two treatment groups, and with a low response rate in both groups.
The company felt as though maybe they needed to go back to the drawing board and look at what happened, because that was certainly not what they had projected, or what anyone had projected, and certainly wasn't our experience in either Toronto or Atlanta. It wasn't the experience in Germany, or in Spain, or in Calgary where other groups were doing small studies. The interesting thing about the industry trial is that while they were focused on an end point for FDA approval at 6 months, patients continued to receive DBS, and did much better with longer stim, with a near$50 \%$ response rate of patients reaching 2 years. There are in fact a group of subjects from the study that continue to receive DBS, despite the closing down of the formal trial; others were explanted. That seems to suggest that while the formal trial failed, the treatment did not-but that will require further study to prove it. My group in Atlanta and now in NY has NIH funding to continue our work using prototype devices that allow recording of local field potentials from the DBS lead during active stimulation, providing a window into the time course of recovery with ongoing DBS treatment. That said, next steps toward potential commercialization by industry are not clear, so that patients are in a limbo unless you're in an experimental study.

Sébastien Thuault: You were recently appointed as director of a new center at the Icahn School of Medicine, the Advanced Circuit Therapeutics Center. Can you tell us about what you're going to do, and from which other angle you're going to approach this terrible disease.

Dr. Mayberg: The opportunity I've been given at Mount Sinai is to build a new center. It's not from scratch, but it's to embed a research group within the clinical neuromodulation team. That's not a new concept, because every functional neurosurgeon or DBS neurologist or psychiatrist has research collaborators. What we're going to do differently is bring a multidisciplinary team of researchers together with DBS clinicians from neurology, neurosurgery, and psychiatry so that movement, mood, and cognitive disorders treated with DBS can be studied in an integrated fashion, hopefully catalyzing opportunities for defining common circuit-tuning strategies for patients regardless of diagnosis.

We have colocalized the clinicians that provide established DBS treatment for movement disorders such as Parkinson's or dystonia or tremor in the same clinical space as the psychiatrists that are using DBS for OCD or doing experimental DBS for any disorder. My depression research group will live alongside clinical movement disorder specialists, and we will build a shared platform for measuring mood, motor, and behavior across diagnoses. We're tuning circuits, broadly; we're not treating specific syndromes or disorders. It's an artificial separation. We are excited and grateful to have across the board support from the institution, the hospital, and the departments for our mission. "We don't want to keep these activities in separate silos; we're going to move everyone together," I'll be able to look at nonmotor features of Parkinson's. Where they treat the motor problems exquisitely well, they do little for apathy, emotional instability, compulsive disor- 
ders that are part of the disorder and can occur as side effects of DBS itself. Similarly, in depression, one of the most striking things we see is that emotion locks down your ability to move. There's actually more alike between a Parkinsonian patient and a profoundly ill depressed patient than we've previously considered, and if we look at them experimentally through the same lens, it may be mechanistically informative and scientifically efficient. I think we can really learn from the different syndromes studied together. Facial expression in depression is profoundly abnormal; it is in movement disorder, as well. Voice, speech, and gait are similarly affected in both dis- orders. By examining all patients with a common set of metrics, we can share knowledge across diagnoses in a new way, so when we get new devices for Parkinson's we can be thinking about how they might be applied in psychiatry. Further, as next-generation devices now allow you to do more than one thing at the same time, maybe we can evolve to where we treat mood and motor dysregulation in Parkinson's off the same electrodes with different contacts and different stimulation parameters. We will work to develop technology innovations that lead to clear clinical innovations. We'll have partners, and it'll be fun and hopefully impactful. 


\section{$\$_{\text {CSH\& }}^{\infty}$ Cold Spring Harbor Symposia SYMPOSIA On Quantitative Biology}

\section{A Conversation with Helen Mayberg}

Cold Spring Harb Symp Quant Biol 2018 83: 264-267 originally published online March 18, 2019 Access the most recent version at doi:10.1101/sqb.2018.83.037598

Creative This article is distributed under the terms of the

Commons http://creativecommons.org/licenses/by-nc/4.0/, which permits reuse and

License redistribution, except for commercial purposes, provided that the original author and source are credited.

Email Alerting Receive free email alerts when new articles cite this article - sign up in Service the box at the top right corner of the article or click here. 